\title{
Medullary hemangioblastoma in a child with von Hippel-Lindau disease: vascular tumor perfusion depicted by arterial spin labeling and dynamic contrast-enhanced imaging
}

\author{
Hyun Woo Goo, MD, PhD, ${ }^{1}$ and Young-Shin Ra, MD, PhD² \\ 1Department of Radiology and Research Institute of Radiology, and 2Department of Neurosurgery, University of Ulsan College of \\ Medicine, Asan Medical Center, Seoul, South Korea

\begin{abstract}
Medullary hemangioblastoma is very rare in children. Based on small nodular enhancement with peritumoral edema and without dilated feeding arteries on conventional MRI, hemangioblastoma, pilocytic astrocytoma, oligodendroglioma, and ganglioglioma were included in the differential diagnosis of the medullary tumor. In this case report, the authors emphasize the diagnostic value of arterial spin labeling and dynamic contrast-enhanced MRI in demonstrating vascular tumor perfusion of hemangioblastoma in a 12-year-old boy who was later found to have von Hippel-Lindau disease.
\end{abstract} \\ http://thejns.org/doi/abs/10.3171/2014.12.PEDS14609
}

KEY WORDS hemangioblastoma; medulla; perfusion MRI; arterial spin labeling; dynamic contrast-enhanced imaging; von Hippel-Lindau disease; oncology

$\mathrm{H}$ EMANGIOBLASTOMA of the CNS is a rare benign tumor of vascular origin, primarily occurs in the 3 rd to 5 th decades of life, and occurs most commonly in the cerebellum. Thus, hemangioblastoma in children, particularly those involving regions other than the cerebellum and spinal cord (such as the brainstem and the cerebral hemispheres), is quite uncommon. Von HippelLindau (VHL) disease is associated with approximately $10 \%$ of patients with hemangioblastoma. The patients with VHL disease have retinal capillary hemangioma and tend to have multiple hemangioblastomas of the CNS, multiple cysts, and tumors in the pancreas, liver, kidney, and lung. The typical MRI finding of hemangioblastoma is described as a cystic mass with a small, vascular, intensely enhancing mural nodule. However, a solid hemangioblastoma is not uncommon, particularly in small, asymptomatic tumors. If present, serpentine flow voids in the solid portion of the tumor on MRI may strongly suggest the diagnosis.

Perfusion MRI demonstrating tumor vascularity is one of the advanced MRI techniques commonly used for evaluating brain tumors. ${ }^{2}$ Dynamic susceptibility-weighted contrast-enhanced (DSC) imaging has been used for differentiating various tumor types by using the relative cerebral blood volume (rCBV) ratio. ${ }^{2-5}$ Perfusion MRI can also be obtained without the injection of an exogenous contrast agent using the arterial spin labeling (ASL) technique. ${ }^{6-8}$ ASL imaging can provide measurements of blood flow in brain tumors. Dynamic contrast-enhanced (DCE) imaging has been recently used principally for evaluating the permeability of brain tumors by using the transfer constant (Ktrans), rate constant (Kep), interstitial volume (Ve), and blood plasma volume (Vp) values. ${ }^{2}$

Exceedingly higher vascular perfusion has been demonstrated with DSC and ASL MRI in adult patients with hemangioblastoma. ${ }^{4-7}$ However, to the best of our knowledge, this feature has not been reported in children with hemangioblastoma. Therefore, in this paper we report on a child with a hemangioblastoma involving the medulla oblongata, in whom highly increased tumor perfusion de-

ABBREVIATIONS ASL = arterial spin labeling; DCE = dynamic contrast-enhanced; DSC = dynamic susceptibility-weighted contrast-enhanced; EPI = echo-planar imaging; $\mathrm{Kep}=$ rate constant; Ktrans = transfer constant; rCBV = relative cerebral blood volume; rTBF = relative tumor blood flow; Ve = interstitial volume; VHL = von Hippel-Lindau; $\mathrm{Vp}=$ blood plasma volume.

SUBMITTED November 4, 2014. ACCEPTED December 17, 2014.

INCLUDE WHEN CITING Published online April 17, 2015; DOI: 10.3171/2014.12.PEDS14609.

DISCLOSURE The authors report no conflict of interest concerning the materials or methods used in this study or the findings specified in this paper. 
picted by ASL and DCE imaging was useful for distinguishing it from other pediatric medullary tumors with similar imaging findings on conventional brain MRI.

\section{Case Report}

History and Examination

A previously healthy 12-year-old boy presented with generalized tonic-clonic seizures. On admission, the patient complained of a tingling sensation on his right thigh, which was gradually improved. A neurological examination and laboratory tests were unremarkable. A T1 hypointense and T2 hyperintense mass, approximately $2.4 \mathrm{~cm}$ in size, with a poorly defined margin was identified in the right posterolateral aspect of the lower medulla on brain MRI (Fig. 1A and B). The mass showed increased water diffusion on the apparent diffusion coefficient map, and no hypointense signals suggesting hemorrhage, neovascularity, or dilated vessels on susceptibility-weighted imaging. Single-voxel proton MR point-resolved spectroscopy obtained in the mass revealed no identifiable metabolites. Two MRI techniques, including ASL and DCE imaging, were performed to evaluate tumor vascularity and vascular permeability in this patient. However, DSC imaging was not performed, because low diagnostic yield resulting from pronounced artifacts and geometrical distortion involving the small medullary lesion was anticipated. Noncontrast perfusion imaging using a pseudo-continuous ASL method was acquired with a single-shot gradient-echo echo-planar imaging (EPI) technique (labeling duration $1650 \mathrm{msec}$, post-label delay $1700 \mathrm{msec}$, TR/TE $4130 / 14 \mathrm{msec}$, flip angle $90^{\circ}$, number of signal averages 1 , SENSE [sensitivity encoding] 2.5 , FOV $220 \times 220 \mathrm{~mm}$, matrix $90 \times 90$ pixels, slice thickness $6 \mathrm{~mm}$, number of slices 19, acquisition time 4 minutes 15 seconds). The perfusion images, generated by subtracting the labeled image from the nonlabeled image at each slice position, showed well-defined round hyperintensity in the medullary lesion comparable to the signal intensities of the internal carotid arteries, indicating highly increased blood flow in
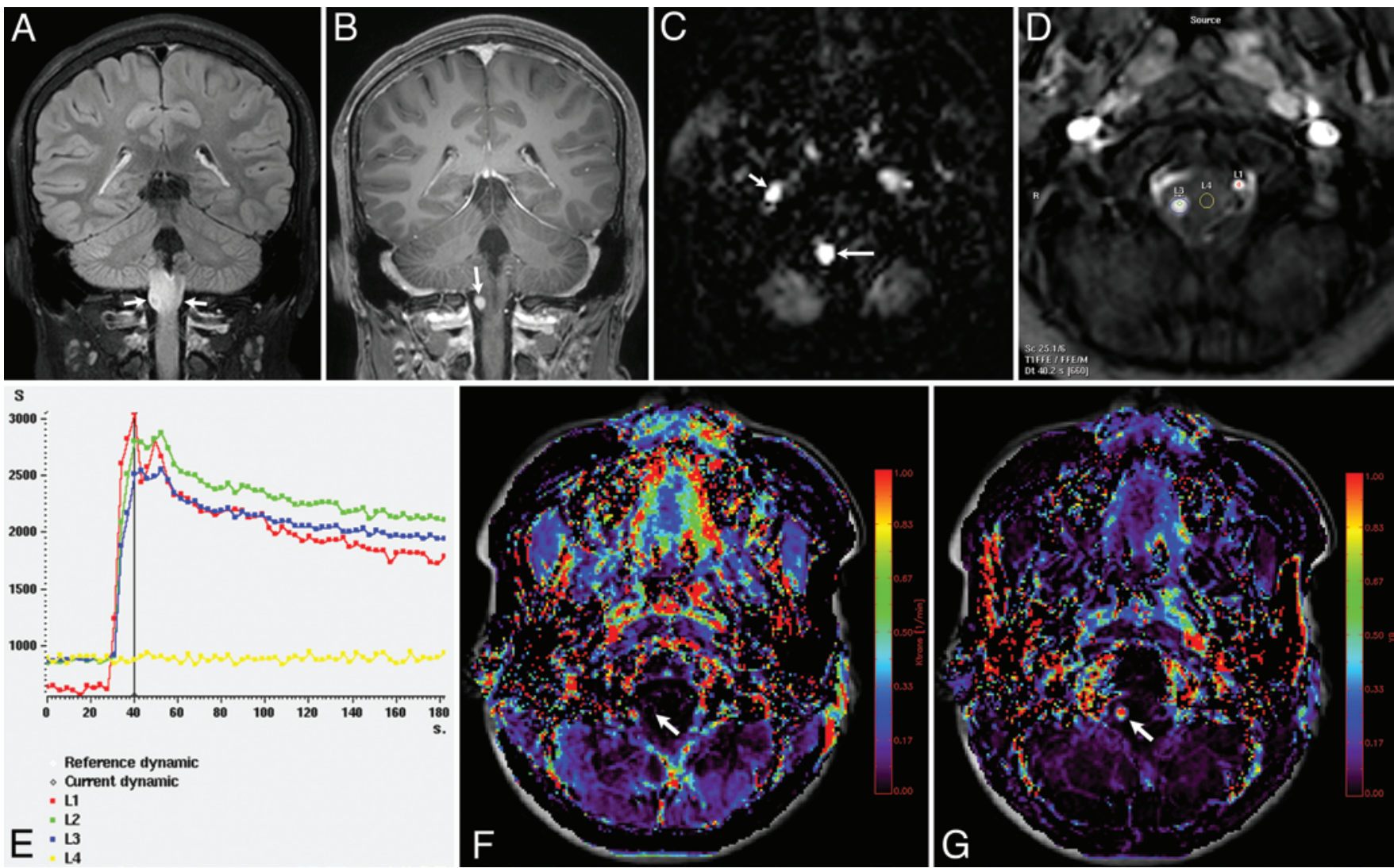

FIG. 1. Imaging findings in the patient. A coronal reformatted FLAIR MR image (A) reveals ill-defined hyperintensity of the lesion, central to right (arrows). A coronal contrast-enhanced T1-weighted MR image (B) demonstrates a well-defined small nodular enhancement (arrow) in the right side of the lesion. An axial pseudo-continuous ASL image (C) shows a small, round, hyperintense lesion (long arrow) in the right posterolateral aspect of the lower medulla comparable to the signal intensity of the right internal carotid artery (short arrow), indicating vascular tumor perfusion (rTBF ratio 11.8). An axial image (D) obtained during the arterial phase of DCE imaging shows the locations of 4 regions of interest: L1, the left vertebral artery; L2, the center of the nodular enhancing lesion; $L 3$, the nodular enhancing lesion; and L4, the normal-appearing medulla. A time-intensity curve (E) demonstrates that the curve pattern of the enhancing lesion ( $L 2$ and $L 3$ ) is almost identical to that of the left vertebral artery (L1). The measured values in the enhancing lesion (arrow) were very low $\left(-0.9 \pm 1.8 \mathrm{~min}^{-1}\right)$ on the Ktrans map (F) and very high $(1.3 \pm 0.9$, arbitrary unit) on the Vp map (G), indicating negligibly low vascular permeability and highly increased blood plasma volume. Figure is available in color online only. 
the tumor (Fig. 1C). The relative tumor blood flow (rTBF) ratio (relative to the contralateral normal-appearing cortical gray matter) of the tumor from ASL imaging was approximately 11.8 . A standard dose $(0.1 \mathrm{mmol} / \mathrm{kg})$ of gadoterate meglumine (Dotarem, Guerbet) was injected intravenously at an injection rate of $3 \mathrm{ml} / \mathrm{sec}$ for postcontrast MRI. DCE imaging was acquired with a T1-weighted 3D gradient-echo sequence (TR/TE $15 / 3 \mathrm{msec}$, flip angle $15^{\circ}$, number of signal averages 1 , FOV $220 \times 220 \mathrm{~mm}$, matrix $224 \times 224$ pixels, slice thickness $6 \mathrm{~mm}$, number of slices 11 , number of dynamic phases 60 , acquisition time 3 minutes). The same pulse sequence with a flip angle of $5^{\circ}$ was performed immediately before the administration of contrast agent to obtain T1 mapping. As in ASL imaging, DCE imaging and time-intensity curve analysis also demonstrated vascular perfusion in the enhancing lesion comparable to those of the vertebral arteries (Fig. 1D and E). DCE-MRI parameters, including Ktrans, Kep, Ve, and $\mathrm{Vp}$ values measured in the enhancing lesion, were -0.9 $\pm 1.8 \mathrm{~min}^{-1}, 0.6 \pm 1.4 \mathrm{~min}^{-1},-0.5 \pm 0.9$ (arbitrary unit), and $1.3 \pm 0.9$ (arbitrary unit), respectively (Fig. 1F and G). An intense nodular enhancement approximately $0.8 \mathrm{~cm}$ in size was shown in the mass lesion on contrast-enhanced T1-weighted MRI (Fig. 1B). The T1- and T2-prolonged area outside the nodular enhancement was regarded as peritumoral edema or a nonenhancing portion of the tumor. Based on the MRI findings, a low-grade tumor such as a hemangioblastoma, pilocytic astrocytoma, oligodendroglioma, or ganglioglioma was suggested. Among the differential diagnoses, hemangioblastoma was the most probable because the mass showed highly increased perfusion and low vascular permeability on ASL and DCE imaging. On the other hand, dilated serpentine feeding arteries, another characteristic MRI finding of hemangioblastoma, were not demonstrated.

\section{Operation and Postoperative Course}

At surgery, a well-defined, pinkish-gray mass with prominent vessels was identified at the right posterolateral side of the lower medulla (Fig. 2 left). Gross-total removal of the mass was performed without any complications. On microscopic examination, the diagnosis of hemangioblastoma was made by identifying the typical histological findings of a rich and tightly packed capillary network in a stroma of clear vacuolated cells (Fig. 2 right).
After surgery, an ophthalmological examination disclosed a retinal capillary hemangioma on the right side in this patient. A history of retinal capillary hemangioma was also documented in the patient's mother and maternal aunt. Abdominopelvic CT and whole-spine MRI were performed to evaluate the involvement of VHL disease in other organs and were unremarkable. A genetic study using polymerase chain reaction sequencing and multiplex ligation-dependent probe amplification eventually confirmed VHL disease in this patient by identifying a VHL gene mutation (c.208G > A [p.Glu70Lys]).

The patient recovered well without any complications and showed stable clinical follow-up results without seizures or focal neurological deficits for 1 year 7 months. His retinal capillary hemangioma was also unchanged on follow-up ophthalmological examinations.

\section{Discussion}

Perfusion MRI has been used to demonstrate tumor vascularity and angiogenesis noninvasively with good correlations with histological assessments. ${ }^{1,6}$ As a result, the imaging technique can be used to differentiate brain tumors with high tumor vascularity from those with low tumor vascularity. ${ }^{2-5}$ In this regard, a hemangioblastoma showing significantly higher vascular perfusion (rCBV ratio 7.7-11.4) can be accurately distinguished from other posterior fossa tumors with similar imaging findings on conventional MRI, such as metastases (rCBV ratio 5.3) and pilocytic astrocytoma (rCBV ratio 1.8), by using DSC MRI in adult patients. ${ }^{4,5}$ Although DSC imaging has been most commonly used for evaluating brain tumor perfusion, it is noteworthy that the pulse sequence using a single-shot gradient-echo EPI technique is vulnerable to magnetic susceptibility artifacts and image distortion, particularly pronounced at the brain-bone-air interfaces or the posterior fossa. Such degraded image quality affecting the results of DSC MRI is especially problematic in pediatric patients because pediatric brain tumors are commonly located in the posterior fossa. Consequently, DSC imaging was not performed in our case with medullary hemangioblastoma. Instead of DSC imaging, pseudo-continuous ASL and DCE imaging were performed to evaluate tumor vascularity and permeability in our case.

As in DSC imaging, the rTBF ratio measured by ASL
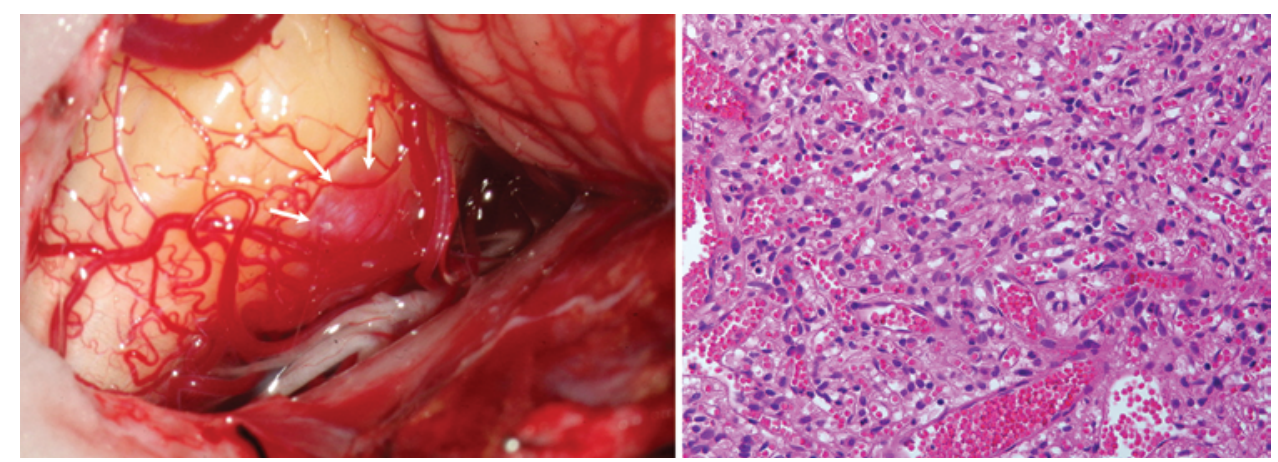

FIG. 2. Surgical and histopathological findings. An intraoperative photograph (left) shows dilated vessels over a well-defined pinkish-gray tumor (arrows). A photomicrograph (right) demonstrates a proliferation of capillaries and large, neoplastic stromal cells with foamy cytoplasm and hyperchromatic nuclei. $\mathrm{H} \& \mathrm{E}$, original magnification $\times 100$. Figure is available in color online only. 
imaging in adults was significantly higher in hemangioblastoma than in metastases $(8.0 \text { vs } 3.0, \mathrm{p}<0.05)^{7}$ and in other brain tumors including gliomas, meningiomas, and schwannomas. ${ }^{7}$ Only a metastasis from renal cell carcinoma showed a very high rTBF ratio (16.2) comparable to that of hemangioblastoma. ${ }^{7}$ In our case, increased blood flow in the tumor on ASL imaging was almost vascular, not only according to the visual assessment, but also by the quantitative assessment (rTBF ratio 11.8), which was quite useful in suggesting hemangioblastoma as the most probable diagnosis of the tumor. ASL imaging is regarded to be particularly advantageous in children because it does not require the intravenous injection of a contrast agent at a high injection rate that is usually necessary for quality DSC imaging and it has fewer susceptibility artifacts than DSC imaging. It should be noted that all hemangioblastomas evaluated with DSC or ASL imaging have been reported in adults but not in children. ${ }^{4-7}$

In a review article, ${ }^{2}$ the time-intensity curve pattern of DCE imaging in the solid portion of the tumor was almost identical to that in the dural venous sinus in an adult with cerebellar hemangioblastoma, as in our case. In the same case, the authors also described that the permeability map of DCE imaging showed significantly high permeability in the solid portion of a cerebellar hemangioblastoma. ${ }^{2}$ However, the latter description is probably incorrect because hemangioblastoma characteristically shows very high vascularity and low vascular permeability, actually proven by high Kep and Vp values and low Ktrans and Ve values in our case. Because we have only limited experience with DCE imaging in hemangioblastoma, we need further evidence to confirm this low vascular permeability initially described in our case.

Compared with pilocytic astrocytomas showing no or minimal peritumoral edema, hemangioblastomas tend to demonstrate moderate to severe peritumoral edema on conventional MRI. ${ }^{5}$ Our case also showed considerable peritumoral edema. In contrast, serpentine flow voids on MRI, one of the characteristic findings of hemangioblastoma, were not identified, even on susceptibility-weighted imaging in our case. As a result, preoperative angiography or embolization was not performed. However, dilated vessels were recognized around the tumor at surgery, which was deemed responsible for vascular tumor perfusion on ASL and DCE imaging. A clinical history of VHL disease was not established at initial presentation in our case, which complicated the preoperative diagnosis of the medullary tumor. After histological confirmation of the medullary tumor, we found a retinal capillary hemangioma in the patient as well as a family history of retinal capillary hemangioma. Finally, the association of medullary hemangioblastoma with VHL disease was confirmed by a genetic study in this patient.

In conclusion, although medullary hemangioblastoma rarely occurs in children, we could demonstrate that vascular tumor perfusion depicted by ASL and DCE imaging was very useful for distinguishing hemangioblastoma from other pediatric medullary tumors with similar conventional MRI findings (such as pilocytic astrocytoma, oligodendroglioma, and ganglioglioma) in a child with VHL disease.

\section{References}

1. Aronen HJ, Gazit IE, Louis DN, Buchbinder BR, Pardo FS, Weisskoff RM, et al: Cerebral blood volume maps of gliomas: comparison with tumor grade and histologic findings. Radiology 191:41-51, 1994

2. Brandão LA, Shiroishi MS, Law M: Brain tumors: a multimodality approach with diffusion-weighted imaging, diffusion tensor imaging, magnetic resonance spectroscopy, dynamic susceptibility contrast and dynamic contrast-enhanced magnetic resonance imaging. Magn Reson Imaging Clin N Am 21:199-239, 2013

3. Cha S: Dynamic susceptibility-weighted contrast-enhanced perfusion MR imaging in pediatric patients. Neuroimaging Clin N Am 16:137-147, ix, 2006

4. Hakyemez B, Erdogan C, Bolca N, Yildirim N, Gokalp G, Parlak M: Evaluation of different cerebral mass lesions by perfusion-weighted MR imaging. J Magn Reson Imaging 24:817-824, 2006

5. Kumar VA, Knopp EA, Zagzag D: Magnetic resonance dynamic susceptibility-weighted contrast-enhanced perfusion imaging in the diagnosis of posterior fossa hemangioblastomas and pilocytic astrocytomas: initial results. J Comput Assist Tomogr 34:825-829, 2010

6. Noguchi T, Yoshiura T, Hiwatashi A, Togao O, Yamashita K, Nagao E, et al: Perfusion imaging of brain tumors using arterial spin-labeling: correlation with histopathologic vascular density. AJNR Am J Neuroradiol 29:688-693, 2008

7. Yamashita K, Yoshiura T, Hiwatashi A, Togao O, Yoshimoto $\mathrm{K}$, Suzuki SO, et al: Arterial spin labeling of hemangioblastoma: differentiation from metastatic brain tumors based on quantitative blood flow measurement. Neuroradiology 54:809-813, 2012

8. Yeom KW, Mitchell LA, Lober RM, Barnes PD, Vogel H, Fisher PG, et al: Arterial spin-labeled perfusion of pediatric brain tumors. AJNR Am J Neuroradiol 35:395-401, 2014

\section{Author Contributions}

Conception and design: Goo. Acquisition of data: Goo. Analysis and interpretation of data: Goo. Drafting the article: Goo. Critically revising the article: both authors. Reviewed submitted version of manuscript: both authors. Approved the final version of the manuscript on behalf of both authors: Goo. Administrative/ technical/material support: both authors. Study supervision: Goo.

\section{Correspondence}

Hyun Woo Goo, Department of Radiology and Research Institute of Radiology, University of Ulsan College of Medicine, Asan Medical Center, 88, Olympic-ro 43-gil, Songpa-gu, Seoul 138736, Korea. email: hwgoo@amc.seoul.kr. 